\title{
EL HOMBRE Y SUS FANTASMAS. SOBRE UNA PROPUESTA DE LA DISECCIÓN ANALÍTICA DE LA NARRATIVA DE ANTONIO MUÑOZ MOLINA Y JAVIER MARÍAS: WIOSNA SZUKAŁA, MESKOŚĆ I FANTAZMAT W PROZIE JAVIERA MARÍASA I ANTONIA MUNOOZA MOLINY. POZNAŃ: WYDAWNICTWO NAUKOWE UAM, 2019
}

La monografía Męskość i fantazmat w prozie Javiera Maríasa i Antonia Muñoza Moliny (Masculinidad y construcciones fantasmáticas en la narrativa de Javier Marías y Antonio Muñoz Molina ${ }^{1}$ ) de Wiosna Szukała (Poznań, 2019) es una de las propuestas que abren paso a un conocimiento más profundo de la narrativa española actual en el ámbito polaco. Escrito en la lengua polaca, con un rigor investigador impresionante, el análisis se centra en la lectura de obras escogidas de Javier Marías y Antonio Muñoz Molina contextualizada en torno a dos nociones fundamentales para la Autora: la masculinidad y el fantasma. Ambos términos -no sólo aquí, sino cada vez que aparecen en todo tipo de estudios- requieren una explicación amplia en cuanto a su alcance semántico y pragmático, sobre todo si se los convierte en herramientas metodológicas y ejes interpretativos de una investigación interdisciplinar, tal como lo hace en su libro Wiosna Szukała.

El fantasma presentado por la Autora (que sigue aquí el pensamiento lacaniano) se entiende como una especie de proyección inalcanzable de los deseos y roles de un yo masculino; como un andamiaje mental sobre el cual se monta un autorretrato al que se aspira y al que, a la vez, se renuncia. La dimensión paradójica de los fantasmas masculinos presentes en las novelas de Antonio Muñoz Molina y Javier Marías le sirve a la investigadora de punto de partida para adentrarse en la complejidad de los protagonistas cuyas vicisitudes, en la mayoría de los casos, se parecen a una caminata incesante por las redes de sus propios pensamientos. Las "aventuras" descritas por ambos autores van dirigiéndose sobre todo hacia el autoconocimiento de los yoes inventados y sus mundos interiores, hacia los destellos de la identidad "verdadera" suya. Por ello, Wiosna Szukała decide alejarse del marco social e histórico ${ }^{2}$ e indica el objetivo de sus consideraciones como el intento de exponer la pluridimensionalidad y ambigüedad en que se estriba la construcción psicológica de los protagonistas tanto de Marías como de Muñoz Molina.

\footnotetext{
${ }^{1}$ La traducción del título no viene de la autora; en el libro no se ofrece ninguna descripción del contenido en la lengua castellana.

${ }^{2}$ Aunque, por supuesto, no es posible hacerlo por completo y, en algunas ocasiones, p.ej. en el fragmento dedicado a Carlota Fainberg, la Autora asocia la crisis de la identidad del protagonista con la transitoriedad y confusión del cambio político-social. (p. 147)
} 
En dos primeros capítulos que constituyen la base teórica de la investigación, la Autora fundamenta su pesquisa metodológica en la corriente psicoanalista y un amplio abanico de fuentes dedicadas a los estudios masculinos. Los enfoques de -entre otros-Sigmund Freud, Jacques Lacan, Raewyn Connell, Judith Butler, Michael Kimmel o Gunnar Karlsson nos permiten repasar la evolución del concepto de la masculinidad y concebirla como una noción teórica y ontológica. La parte dedicada a lo masculino en la reflexión humanística constituye una muestra alucinante de la soltura con la cual Szukała se mueve por los laberintos terminológicos y conceptuales derivados de los trabajos tanto clásicos como más recientes. Lo único que puede sorprender aquí es poca relevancia que se da a las aportaciones españolas con respecto a masculinity studies, sobre todo porque sabemos que la problemática de la feminidad y masculinidad en España (también en relación con la "glotofagia" o "invisiilidad" entendida a modo rancieriano) gana cada vez más interés entre los representantes de distintas disciplinas, asimismo de los estudios literarios (la misma Autora lo menciona de paso en una nota a pie de página; p. 11). A lo mejor valdría la pena ofrecer a los lectores polacos un recorrido por las propuestas que -les guste a Marías y Muñoz Molina o no- provienen de su "primera patria".

El siguiente capítulo corrobora la utilidad metodológica del ya mencionado concepto del fantasma. El fantasma une par excellence lo masculino con lo ficticio, puesto que exhibe lo masculino precisamente como una construcción fantasmática, una work in progress perpetua, una aspiración y no tanto como una característica dada y poseída, inscrita en la identidad de los hombres por el simple hecho de ser un varón. En consecuencia de ese estatus efímero, la voz de lo masculino necesita ser (re)creada, (re)pensada y recuperada, para lo cual se muestra muy eficaz el procedimiento de construir narraciones, tanto colectivas como individuales, así pues, efectivamente, la literatura. Szukała se deja ver otra vez como una lectora muy atenta a las fluctuaciones terminológicas y un repertorio amplio de perspectivas dedicadas al fantasma en el nivel interdisciplinar, si bien como los pilares teóricos escoge en el tercer capítulo las consideraciones de Sigmund Freud, Slavoj Žižek, Jacques Lacan y Kaja Silverman. La investigadora americana encuentra un lugar relevante en las indagaciones de Szukała, dado que por medio de las referencias a la equiparación colectiva de la masculinidad con el falo y la importancia de la Ley de Padre manifestada en la proyección del ideal de lo masculino que rige el Orden Simbólico (conforme la terminología lacaniana), Silverman hace hincapié en la existencia de las articulaciones de la masculinidad que se alejan de la "ficción dominante" erigida en torno al orden falogocéntrico. Silverman habla de las articulaciones que admiten una masculinidad perversa, desviada, "anómala", entre las cuales Szukała halla rasgos constitutivos de la identidad de los protagonistas de novelas analizadas. Protagonistas, añadamos, que sufren por las limitaciones del orden tradicional, patriarcal, repetitivo, que les quiere quitar una dosis tremenda de la libertad, desenvoltura e independencia sin la cual se ahogan.

Con la denuncia del orden falogocéntrico y, por consiguiente, con el último eslabón metodológico expuesto, pasamos a un esbozo histórico-literario que revela ante los lectores polacos el lugar de Antonio Muñoz Molina y de Javier Marías en el panorama de la narrativa española del siglo XX y XXI. Es un capítulo de gran importancia, ya que a pesar de que se trate de los autores traducidos al polaco, en la mayoría de los casos, los no hispanistas pueden aprovecharse de algún tipo de introducción para contextualizar la lectura de las novelas escogidas y analizadas por la Autora. Encontramos aquí informaciones sobre el estilo individual de ambos prosistas, la internacionalización de su estatus en el mercado literario, la importan- 
cia del cine y alusiones intertextuales en su narrativa, así como las características del protagonista modélico de Muñoz Molina y Marías.

La parte siguiente se dedica ya a las interpretaciones de los textos concretos de ambos novelistas. Empezando por Marías, se indica que el núcleo temático de su narrativa, según Manuel Alberca, es la pérdida de la identidad y el intento de reencontrarla: un proceso duradero, nada fácil, si bien fascinante, que refleja los mecanismos que permiten a un yo masculino sobrellevar los momentos dificultosos de la crisis existencial. Szukała reconoce el peso de ello mostrando comentarios y autocomentarios explícitos de los mismos protagonistas, verbigracia, de Todas las almas. Con la intención de resumir la condición del yo narrativo creado por Marías, con toda la incertidumbre e imposibilidad de consolidar su autoimagen, la Autora cita a Alberca: «Es un personaje contradictorio a la fuerza, pues sugiere o expresa tanto su falta de entidad como la necesidad obsesiva de afirmación. Es como si la debilidad de su yo se fortaleciese en cada acción o sentencia verbal. Este protagonista hace ostentación de su vulnerabilidad, de su soledad y de sus perturbaciones, y no contento con reconocerlas, las pone en escena» (p. 99).

La inclusión de estudios de varios historiadores y críticos procedentes del ámbito hispanohablante (no solo de Alberca, sino también de Domingo Rodenas de Moya, José Carlos Mainer o Teresa González Arce, a los que mencionamos solamente a modo de ejemplo) la vemos como una invitación para los lectores polacos a conocer el pensamiento históricoliterario contemporáneo hispano, raramente llevado en otras ocasiones al contexto polaco (no obstante, lo popularizan también los trabajos de, entre otros, Magda Potok, Justyna Ziarkowska, Piotr Sawicki, Arkadiusz Żychliński o Łukasz Smuga). Es fenomenal que la Autora cite a varios investigadores, también algunos del mundo anglosajón, de modo bilingüe, porque gracias a ello el apetito epistemológico (y terminológico) de los que conocen más de una lengua (lo cual, suponemos, es hoy en día un hecho) crece y les anima a profundizar los temas abordados incluso más.

La bibliografía, sin duda alguna, es extensa, aunque se podría incluir en ella también algunas publicaciones de José María Pozuelo Yvancos, verbigracia, su Novela española del siglo XXI (Cátedra, 2017), donde el autor dedica un capítulo extenso al «ciclo de Oxford» y Los enamoramientos de Javier Marías: novelas que son objetos de investigación de la investigadora polaca. Pozuelo Yvancos asimismo pone de relieve las características de la literatura ensimismada escrita por Marías en Figuraciones del yo en la narrativa. Javier Marías y E. Vila-Matas (Universidad de Valladolid, 2010), así que también este título podría encontrar un lugar en el corpus bibliográfico de Wiosna Szukała.

Tampoco se menciona un libro de Marta Pérez-Carbonell: The Fictional World of Javier Marias. Language and Uncertainty (Brill, 2016) que podría haber sido de interés para la Autora, puesto que Pérez-Carbonell investiga detalladamente el asunto del estilo de Marías, centrándose en la incertidumbre, el silencio y la omisión, la traducción (y autotraducción), así como en el impacto de lo contado en la dimensión real: «[T]he opening line of Rostro: 'No debería uno contar nunca nada, ni dar datos ni aportar historias ni hacer que la gente recuerde a seres que jamás han existido ni pisado la tierra o cruzado el mundo' (FL, 13) draws attention to the extent to which language may affect the reality: whether it refers to real stories ('dar datos') or fictional ones ('hacer que la gente recuerde a seres que jamás han existido'), language has very obvious consequences on reality and people's lives. If one accepts that language has the power to affect reality whether it recounts real or fictional stories, fiction 
itself would have the power to affect reality too, which is why Logie, when analyzing Marías's fiction, invites readers to 'considerar la literatura una práctica performativa'» (Pérez-Carbonell, 2016, p. 18). Citamos este fragmento, debido a que los "valores performativos" tanto del fantasma como del idioma que expresa lo masculino desempeñan un papel importante en las interpretaciones de Szukała y, evidentemente, en el proceso de la construcción de la subjetividad de los protagonistas en las novelas de ambos autores, también de Muñoz Molina.

Wiosna Szukała pone énfasis en la grieta que caracteriza el estado anímico de los yoes masculinos novelescos: un estado que se sitúa entre la pasividad y la pretensión de alcanzar algún logro designado por la proyección fantasmática. Los protagonistas, por un lado, no quieren hacer nada (principalmente, nada proveniente de los escenarios socio-culturales dictados por las expectativas de la época), no obstante, por otro lado, sienten un anhelo fuerte de realizar una visión fantasmática de lo masculino y, con ello, acercarse a la (re)creación más coherente de su identidad por medio de la lengua. El idioma constituye en este proceso la herramienta principal del "pensamiento literario" en su vertiente epistemológica. La lengua influye en el modo de manejar la incertidumbre existencial y permite suavizar (hasta cierto punto) el impacto de las dudas (podemos aludir aquí al término de la "estética de la duda" utilizado por Magda Potok a la hora de resumir la estrategia expresiva de Marías). Las consideraciones de Pérez-Carbonell sobre la performatividad del lenguaje, así como las reflexiones de la bipolaridad intrínseca del fantasma probada por Szukała concuerdan con el siguiente enfoque mencionado por la Autora: la perspectiva de Ernst E. Boesch que analiza la estructura narrativa del fantasma dándole el papel de un filtro organizador de la experiencia individual para que el yo consiga cierto nivel de consistencia interna (Szukała, p. 129). Dicha consistencia y orden son esencialmente lo que buscan los protagonistas de Tu rostro mañana o El jinete polaco.

Como vemos, en la parte que debe ser más bien "práctica" e "interpretativa", la Autora sigue enriqueciendo el telón de fondo del pensamiento metodológico en torno a la literatura y la psicología, verbigracia, con Boesch (ya indicado) o con una referencia a Clarence Craaford, un psicoanalista más, con su visión narratológica de lo inconsciente según la cual «Man is a Story». En efecto, Szukała subraya que los autores españoles de la época de la Transición no tienen fe en el lenguaje como tal, sino lo ven como un medio para crear su identidad a través de contar. Por consiguiente, la identidad individual es un producto del cuento, y no al revés (p. 130).

La Autora demuestra que los fantasmas admitidos por uno en sí mismo se acumulan y sobreponen, y, en consecuencia, tergiversan la claridad de la imagen del yo, tal y como ocurre, por ejemplo, en el caso del protagonista de Tu rostro mañana: Jacques Deza. Su talking cure le abre el camino no solo a la autoexpresión y auto(re)creación, sino también pasa a ser una causa incuestionable de la ansiedad y del desasosiego existencial que Deza siente con respecto a la posibilidad de desenrollar una narración equivocada, falsa. Las consecuencias de un procedimiento narrativo erróneo, como se nos indica en Tu rostro..., pueden ser graves y definitivas (p. 131).

Por la teatralidad intrínseca del proceso de contar (todo el yo quiere encontrar a un oyente atento) y la inclinación humana a la imitatio de los caminos de los demás, sean ficticios o no, Szukała señala el coste emocional que acompaña a los yoes masculinos en la búsqueda de su posicionamiento en el mundo. Se trata de la lucha interna que observamos, por ejemplo, 
en el argumento de Carlota Fainberg de Muñoz Molina, en el que Claudio encuentra a su doble: un tipo grosero, ruidoso y chocarrero, en cuya conducta el académico reconoce una encarnación de la españolidad estereotipada. La conversación con su compatriota junto con la proyección fantasmática en la que Claudio quiere repetir las aventuras de Manuel y vivir de forma diferente y apasionada, por acabar en un fracaso total le hace darse cuenta de que no cumple con las expectativas en ningún ámbito importante: ni profesional, ni social. Empieza a notar que no se siente bien en España ni en el extranjero, que no es tan progresista y liberal como pensaba, que no sabe manejar las condiciones para recuperar un yo fuerte y apasionado (arquetípicamente masculino), aún ensimismado, sensible y refinado (masculino à rebours). El aura misteriosa del encuentro de Claudio y Marcelo es paralela al juego al que invita Muñoz Molina aquí al lector; según Szukała (p. 148), no podemos estar totalmente seguros del carácter del encuentro relatado, ya que podría transcurrir en el nivel fantasmático, como una proyección de la inquietud e incomodidad de Claudio en el plexo de sus circunstancias. Por medio del encuentro de dos varones diametralmente distintos (empero, de un modo peculiar, inseparables), Antonio Muñoz Molina también crea un espacio para tocar el tema del peso de ser español, un tema igualmente crucial para Marías.

En un artículo dedicado no solo a los mismos autores (Marías y Muñoz Molina), sino incluso a las mismas obras suyas (Todas las almas, Corazón tan blanco y Carlota Fainberg) que ocupan la atención de Szukała, Carolina P. Amador Moreno dice que: «Their stories lack the Spanishness, localism, realism and seriousness that characterized the type of writing produced in the three decades that followed the end of the Civil War [...] [T]heir respective styles are a reaction against the patriotism, mimesis and Spanishness which was so characteristic of previous generations of writing. In their rejection of all those traditional elements, their particular styles place the emphasis on form and language, and open the door to the influence of foreign elements. The incorporation of foreign words and foreign cultural allusions forges the basis of their heterodox» ("Javier Marías y Antonio Muñoz Molina: Between two languages", p. 202). Amador Moreno ve en este "rechazo" de la españolidad la disponibilidad a abrirse a nuevas posibilidades creativas que permiten a los autores «challenge the traditional limits of their own literary medium» (p. 202). El artículo de Amador Moreno, aunque desapercibido por Szukała, se muestra relevante si admitimos la necesidad de matizar el análisis de los juegos con lo extranjero en el nivel intertextual y lingüístico presentes en las novelas analizadas; juegos que, añadamos, contribuyen a la condición camaleónica de los constructos autorreferenciales en los que se reflejan los yoes fantasmáticos de los protagonistas masculinos. A su vez, la Autora prefiere buscar este carácter proteico en el nivel argumental de las obras, aunque el manejo de la lengua (o, más bien, las lenguas, ya que el papel del inglés y de las referencias a la literatura anglosajona no es aquí nada casual) resulta clave en cuanto a la autocreación de los protagonistas, por lo cual no merece la pena situar la cuestión del estilo en el segundo plano de las indagaciones en torno a las estrategias novelescas de ambos escritores.

En los siguientes subcapítulos, Szukała alude a las consideraciones de Slavoj Žižek y Jacques Lacan para interpretar en clave psicoanalítica el motivo de Doppelgänger en La canción de Lord Rendall, un texto que nos permite situar a Marías entre los autores de cuentos fantásticos. La Autora presenta la trayectoria de los protagonistas de El jinete polaco y El dueño del secreto de Muñoz Molina con el fin de hacer hincapié en la incertidumbre adscrita a todas las decisiones individuales e imposibilidad de permear los mundos interiores 
de los demás. Pone de relieve el carácter de los personajes femeninos y de forma humorística (y acertada) los compara con el gato de Schrödinger: existente y ausente al mismo tiempo. La introducción de las figuras femeninas les sirve a los protagonistas sobre todo para entablar una conversación nueva consigo mismo, con su pasado, sus deseos y aspiraciones. La imagen de los personajes femeninos en ambos casos es, más bien, quebradiza; no se suele concretizar cien por cien y se difumina, porque lo único que se necesita en el proceso de entrar en contacto con alguna "ella" es un esbozo de lo femenino, un contorno físico capaz de saciar la necesidad de experimentar la cercanía de la belleza o sentir pasión carnal. El yo masculino en novelas de Muñoz Molina y Marías entabla una relación con un personaje femenino como si participase en un espectáculo conocido de las películas, canciones y relatos de los demás. De este modo, el papel de la mujer es a menudo puramente pragmático: con su intimidad y compañía el fantasma femenino llena un hueco cuya existencia amenaza la consistencia de la identidad de un yo varonil.

En la novelística de Muñoz Molina, la mujer, con la intención de aprovecharse del miedo de la castración por parte del hombre y divertirse un poco a lo largo del proceso amoroso, suele asumir el papel de una femme fatale y, en realidad, tampoco quiere que el hombre penetre el misterio en el que se ve rodeada. A su vez, en los personajes femeninos frecuentemente se manifiesta también "el miedo de la mediocridad" (el término de Olga Lopez-Valero Colbert; p. 168), que caracteriza, de hecho, a la mayoría de los protagonistas masculinos de ambos escritores.

Para evitar la existencia mundana de corte burguesa que es la quintaesencia de la vulgaridad según los narradores de Muñoz Molina (p. 127), o bien para huir del matrimonio concebido como falta de libertad (p. 182), los protagonistas prefieren plantearse otros escenarios existenciales. Al igual que los yoes masculinos de Marías, los de Muñoz Molina adoptan el rol de los intelectuales, son reflexivos y, al fin, asumen deliberadamente la identidad fantasmática de un jugador solitario y ensimismado como un flâneur, un detective, un cowboy, un jinete o un cosmonauta (p. 201). Es una de las advertencias que demuestra el carácter anítetico de los fantasmas que intentan adoptar los protagonistas en las novelas de ambos autores. Intentan y no lo alcanzan, porque, en realidad, entran en una polémica incesante con su deseo de omnipotencia y compleción y, a la vez, huyen de las expectativas a las que se ven sometidos. Con la mirada fijada en dichos fantasmas, analizándolos de modo concienzudo y con soltura, Wiosna Szukała consigue lo que indicaba como el objetivo de sus reflexiones: analizar detalladamente el meollo fantasmático de la identidad de los yoes masculinos en la narrativa de Javier Marías y Antonio Muñoz Molina. 


\section{BIBLIOGRAFÍA}

Alberca, M. (2013). El autor como espía y fantasma (autorretrato emboscado de Javier Marías). Letras de Hoje: Estudos e debates de assuntos de lingüistica, literatura e língua portuguesa, 48, 4, 448-458.

Amador Moreno, C.P. (2005). Javier Marías and Antonio Muñoz Molina: between two languages. Linguistica Antverpiensia, 4, 201-204.

López-Valero Colbert, O. (2007). The Gaze on the Past: Popular Culture and History in Antonio Munoz Molina's Novels. Lewisburg: Bucknell University Press.

Pérez-Carbonell, M. (2016). The Fictional World of Javier Marias. Language and Uncertainty. Leiden-Boston: Brill, Rodopi.

Pozuelo Yvancos, J.M. (2010). Figuraciones del yo en la narrativa. Javier Marías y E. Vila-Matas. Valladolid: Universidad de Valladolid.

Pozuelo Yvancos, J.M. (2017). Novela española del siglo XXI. Madrid: Cátedra.

Silverman, K. (1992). Male subjectivity at the margins. London: Routledge.

Szukała, W. (2019). Męskość i fantazmat w prozie Javiera Maríasa i Antonia Muñoza Moliny. Poznań: Wydawnictwo Naukowe UAM.

Katarzyna Gutkowska-Ociepa Uniwersytet Śląski w Katowicach 